\title{
UPDATE ON THE UNIVERSITY OF TORONTO GRADUATE ATTRIBUTE PROCESS
}

\author{
Susan McCahan and Lisa Romkey \\ University of Toronto \\ mccahan@mie.utoronto.ca, romkey@ecf.utoronto.ca
}

\begin{abstract}
The Faculty of Applied Science and Engineering at the University of Toronto has been working through the development of a continuous curriculum improvement process for the past two years. The main group working on this is the Graduate Attributes Committee (GAC) which is made up of faculty representatives from each department. In this paper and presentation we will describe the process we have developed. In addition, we will show examples of the materials that the GAC has produced. Of particular interest are the extensive rubrics that have been developed that can be used as a starting point for professors tasked with assessing the learning outcomes identified for the Graduate Attributes. Faculty have begun to customize these generic rubrics for particular assignments, and examples will be shown of this work. The development process has resulted in reflection and discussion on our curriculum. The development process has also led to reflection on the difficulties involved in assessing the Graduate Attributes and compiling the data we collect. These issues will be explored briefly in the paper.
\end{abstract}

Keywords: Graduate Attributes, Outcomes, Indicators, Rubrics

\section{INTRODUCTION}

In 2010, the Faculty of Applied Science and Engineering at the University of Toronto created a Graduate Attributes Committee (GAC). The GAC includes representation from the 9 programs and from the other units that support student learning directly, such as the Engineering Communication Program (ECP). The GAC was tasked with managing the development of the continuous curriculum improvement process for the Faculty. This included deciding what parts of the process would be Faculty wide, i.e. consistent across all 9 programs, and what parts of the process would be handled at the departmental level.
The GAC has met frequently for the last two years and made substantial progress, also documented in [2].

The members of the GAC have been responsible for developing a full set of global outcomes and indicators associated with each of the 12 graduate attributes. However, the GAC has also frequently invited other faculty to participate in the process. For example, an early draft of the indicators was vetted by an expert on assessment from the Ontario Institute for the Study of Education (OISE) at the University of Toronto. This feedback helped us refine our indicators. We also made use of faculty in engineering with special interests and areas of expertise, such as our Engineering Communication Program, who worked out the first version of our indicators for the Communication attribute. In total, more than 20 faculty have participated actively in the graduate attribute process to date, and this number will grow as the process is implemented.

This participation rate has added substantially to the resources we have available to successfully implement this system. The GAC members and other faculty and staff who worked with the GAC have developed a level of understanding that will allow them to successfully translate the Faculty materials we have developed into effective, valid instruments for assessing learning outcomes and to interpret the resulting data. The programs will be reporting on their outcomes, interpretation of data, and analysis to the GAC during the implementation phase of the project. This exchange of information and vetting of the results will serve to keep all programs moving forward on implementation and support a high level of quality in the process.

\section{METHODOLOGY}

The methodology used at the University of Toronto follows the process suggested by the Engineering Graduate Attributes Development (EGAD) group, an advisory group to the National Council of the Deans of Engineering and Applied Science. The 5 step process involves: 
1. Program evaluation

2. Curriculum mapping

3. Collecting data on student learning

4. Analyzing and interpreting the data

5. Data informed curriculum improvement

\subsection{Program Evaluation}

The Graduate Attributes Committee (GAC) has spent most of their effort over the last two years on the first step: Program evaluation. This work involved developing a set of global learning outcomes that are common across the Faculty and address all 12 of the graduate attributes. The global outcomes define the competencies that we believe all University of Toronto engineering students should achieve by graduation in the 12 dimensions of the attributes. However, the global outcomes represent fairly high level goals that are not directly measurable. To assess the global outcomes the GAC has also developed a set of measurable indicators. This process and the results are discussed in more detail in section 3.

\subsection{Curriculum Mapping}

Concurrent with the GAC work, each program was tasked with developing a set of curriculum maps (see section 4). These curriculum maps illustrate where material associated with the attributes is taught; where students have an opportunity to practice skills associated with the attributes; and where students demonstrate their competency and are assessed in these dimensions. The curriculum maps are unique for each program. They show the direct formal assessment that we propose to use to collect data on student learning outcomes. They also show the proposed indirect measures that will be used to round out the data set.

\subsection{Collecting Data on Student Learning}

We have taken a two-pronged approach to the data collection process. At the Faculty level, the GAC has developed rubrics for the indicators. The rubrics developed by the GAC are meant to be used as a starting point, or exemplar set, for instructors who will be collecting data for the graduate attribute process. Simultaneously, at the program level, data is being collected in a select set of courses. This pilot data collection project is allowing instructors to get an understanding of the adjustments they will need to make to their assessment methods in order to align the assessment with the outcomes they want to measure. Through this process we have learned that many of the rubrics and other assessment tools we have been using require some modification to improve the validity of the data collection methodology. This step is discussed in more detail in sections 5 and 6 .

\subsection{Analyzing and Interpreting the Data}

We have just begun to analyze the data we have collected. To date, the data collected is very sparse and primarily concentrated on the major design components in the curricula. At this stage in the implementation of the graduate attribute process, the data collection is teaching us more about our collection methodology than producing meaningful results. While we can draw a few preliminary conclusions from the data, we should not over react to the limited results we have at this point. The process of collecting the preliminary data, however, is helping us immensely to further the development of a robust, valid and reliable data collection plan. The data collected to date, and the discussion of the results, is given in section 6 and the appendices.

\subsection{Data Informed Curriculum Improvement}

We have not progressed to the point where we can substantially begin data informed curriculum improvement, although the pilot data has provided departments with some preliminary indications of students' strengths and weaknesses, and the validity and reliability of our assessment methodology. However, we have developed a plan for continuous curriculum improvement. The plan will be discussed in section 7 . Our plan will be implemented in 2012/13 and will result in data that can be used for curriculum reform. Following the analysis of the data, the first significant round of data informed curriculum improvement will occur in 2013/14.

\section{OUTCOMES AND INDICATORS}

The Graduate Attributes Committee (GAC) took a three step approach to the development of the indicators that will be used as the foundation for the continuous curriculum improvement process. In this explanation of the development of our indicators we make reference to “assessment points". These are points in the students' development where we are assessing their proficiency in relation to a particular indicator. The assessment is a measure of the quality of a performance (e.g. oral presentation) or artifact (e.g. written response to an exam question) that demonstrates learning. The assessment point could be direct, such as an exam or lab 
report, or indirect, such as a survey or annual data on academic offense cases.

As a first step, the GAC developed global outcomes based on each attribute. Global outcome is synonymous with "global objectives" as defined by Anderson, Krathwohl, et al [1]. They state "Global objectives are complex, multifaceted learning outcomes that require substantial time and instruction to accomplish. They are broadly stated and encompass a large number of more specific objectives" (p.15). The global outcomes represent our learning priorities for our students, and define broadly the competencies we want our students to achieve by graduation. The global outcomes developed by the GAC are common across all 9 of the programs in the Faculty of Applied Science and Engineering at the University of Toronto. The global outcomes associated with each attribute are shown in Appendix A . We have developed between 2 and 4 essential global outcomes for each attribute, which should be enough for us to identify areas of strength and weakness across the attributes and within each attribute (to inform our curriculum evolution) while avoiding the assessment of so many aspects of each attribute that we create an unsustainably onerous data collection system. The global outcomes are important because they create a shared understanding of the competencies we want our students to achieve. And any program is free to add additional outcomes to this basic list if they wish. However, global outcomes are not typically specific enough to be measured directly. They serve as an intermediate step to creation of measurable indicators.

In the second step, the GAC developed indicators based on the global outcomes that we had defined. There are many educational outcomes, i.e. indicators, that could be associated with each of the global outcomes. To create a meaningful and sustainable data collection methodology we used the concept of leading indicators, which serve to identify key performance indicators of learning for each of the global outcomes. Leading indicators is a term most often associated with economic assessment, however Gloria Rogers [4], an expert in educational assessment, has suggested the concept of leading indicators as a useful way of understanding the role of performance indicators in outcomes based accreditation processes. The GAC developed these indicators, using expert sub-committees and consultation with other Faculty members without reference to a specific program or point of assessment within a given program. The indicators at this level are, therefore, necessarily somewhat general. The GAC also defined more indicators than any one program could, or should, use. The list is not extensive; each indicator is central to the global outcome it purports to measure.
However, giving a range of indicators allows each of the 9 programs to select a subset that best fits with their current courses, extra or co-curricular activities, and assessment methods. A current list of indicators is available from the authors on request.

In the third step, each program needs to select a subset of indicators to use to assess the global outcomes. They are generally choosing from the Faculty list of indicators, and adapting these to fit the specific type of performance they are assessing. For example, a program might pick "Describe the causes of a problem and its effects", from the Faculty list of indicators for Problem Analysis. Suppose they want to apply this indicator to assess student performance on an assignment such as a report on traffic congestion in Toronto written in a third year Civil Engineering course. The program may choose to modify the indicator to read "Describes the causes of traffic congestion in Toronto and its effects" to include as a criteria on their marking rubric. The results from this assessment will indicate to some degree the cohort's "demonstrated ability to identify and characterize an engineering problem" which is the global outcome associated with this indicator. This data, combined with results from other assessment information in this global outcome category, will produce a picture of the achievement level of the cohort on this outcome.

A different program may choose "Classify a given problem, and the type of solution sought" from the same global outcome indicator list. They may, for example, modify this to fit a question asked on a final exam; e.g. "Part a) Given the stated problem, classify this problem as under determined, over determined or indeterminate and identify the type of solution sought." The performance results from this part of this question would be collected to indicate the class' "demonstrated ability to identify and characterize an engineering problem.” Although this is a different indicator than the example from the Civil course, the data indicates the level of proficiency of the students on the same global outcome. Basically, this is two different ways to measure performance quality on the same outcome.

To create a reliable data set each program needs to select a broad set of indicators. The set they choose should cover every outcome. And they need to apply these indicators at several assessment points (preexisting or new) in their program to generate a reliable data set. In CEAB terminology this is referred to as triangulation. If none of the indicators developed by the Faculty GAC suits the assessment point they have chosen, then a program is free to develop another indicator that aligns well with the global outcome. However, this new indicator will be brought up for 
review by the GAC to confirm that it is a leading indicator that is well aligned. Not every outcome can be assessed at every assessment point, for example it is not reasonable to try to assess every outcome based on a final design report in a capstone design course. Assessing too many outcomes within any one assessment piece can negatively impact the reliability and validity of the assessment itself. Therefore it is important to consider both whether the indicator is well aligned with the outcome, and also whether the indicator is well aligned with the assessment (performance or artifact). This review process by the GAC not only ensures the integrity of the indicator list in the Faculty, but also makes the new indicator available to other programs who may find that it fits an assessment point they would like to use for gathering data.

\section{CURRICULUM MAPPING}

Curriculum mapping is a crucial step in a continuous curriculum improvement process. Each program must identify how they support student competency development from the time the student enters the program to the point where they are ready to graduate. In particular, we needed to map the curriculum in the 12 dimensions that represent the graduate attributes so we can identify assessment points, but also so we can identify gaps, overlaps, and points of weakness in the curriculum for improvement purposes.

The 9 programs were asked to develop their curriculum maps. Programs took diverse approaches to this activity, with some using an Introduce, Teach, Utilize (ITU) instructor survey approach to map the global outcomes to the curricula [3]. In addition, points of assessment (A) were noted and described. Some programs also chose to identify "Emphasize" (E) in their program curricula. Emphasize (E) signals a strong focus for a particular outcome, e.g. a course with significant design experience, or where communication content is explicitly taught. Other programs used instructor subcommittees to create curriculum maps that document the assessment of each global outcome at various points in the program.

In some instances, a composite mark is well aligned with an indicator. In particular, the knowledge base attribute lends itself to using a combination of composite marks and partial marks. For example, we can generally assess the strength of our students in the area of basic statics by looking at the average and grade distribution in this course and compare this to how our students perform in first year calculus. This data may help us decide whether our students are meeting our expectations more readily in physics than in math, and inform where we focus our attention to improve the curriculum or teaching. To decide what areas of math our students are struggling with most, we need to look at their performance in more specific detail using partial marks (i.e. a specified set of test questions in a particular area of mathematics). This information allows us to sharpen our curriculum improvements to address more specific weaknesses within the subject field.

The assessment plan consists of selecting a set of the existing assessment points to target for data collection from the curriculum map and adding any additional new or indirect assessments necessary to generate a reliable data set for evaluating cohort performance on each global outcome. In many cases the direct measures will be part of a mark on an assignment or other type of assessment. For example, it could be the mark on one targeted question on an exam. Or it could be one row in a rubric that assesses a specific criterion (indicator), out of several criteria that cut across different attributes being assessed on a report (e.g. various aspects of communication, critical thinking, problem analysis, investigation, etc.). Being selective in choosing the points for data collection helps us keep the data collection process manageable. It also helps us create a consistent monitoring system, like placing weather stations in the curriculum map, where we will ensure that the assessment year-over-year continues to provide data on a particular outcome.

\section{DEVELOPMENT OF RUBRICS AND SUPPORT FOR ASSESSMENT}

Members of the GAC developed a set of rubrics to complement the outcomes and indicators for each of the 12 attributes. These rubrics are developed outside of a specific assessment context, but can be used as a "starting point" for course-based assignment rubrics, and can be adapted to specific assessment pieces.

These rubrics set a common threshold or target (i.e. fails, below expectations, meets expectations, exceeds expectations) for all programs on each indicator, creating consistency between programs, yet are flexible enough to be adapted to different assessment types. It also provides useful scaffolding for assessment pieces that are typically scored using a numerical grading scheme, rather than a rubric. So far, rubrics have been developed for 11 of the 12 attributes. These rubrics are in draft stage and will be shown in the presentation and made available as handouts. 


\section{IMPLEMENTATION OF THE ASSESSMENT PLAN}

So far, all 9 undergraduate programs have collected some pilot data for the Graduate Attributes process. The pilot data collection process has highlighted some important considerations to be made in the assessment process. For example, in the 2010-11 pilot collection, the Engineering Science program used a rubric with clear criteria, which could be mapped to indicators, however the rubric did not provide a description of the four levels of competency for each indicator, which creates problems for a) inter-rater reliability and b) achieving a common understanding of the competency level of students. This has since been modified to improve the reliability of the data.

\section{PLAN FOR CONTINUOUS CURRICULUM IMPROVEMENT}

Our Faculty is implementing a specific cycle to support a continuous assessment and improvement cycle:

- 2012/13: years 1 and 4 -- collect data from any assessment points located in years 1 and 4 of each program

- 2013/14: collect data from any assessment points located in year 2 of each program; and analyze data collected in 2012/13 for use in curriculum improvement; begin curriculum changes based on data.

- 2014/15: collect data from any assessment points located in year 3 of each program; and analyze data collected in 2013/14 for use in curriculum improvement; continue curriculum changes based on data.

- 2015/16: repeat years 1 and 4 collection.

The benefit of this approach is that programs can follow a single cohort of students from year 1 to graduation. Also, the collection and analysis of data are distributed over a reasonable time frame, promoting an ongoing curriculum evaluation and improvement process, rather than something departments and instructors engage in every four years.

\section{CONCLUSIONS}

Through a five-step process, the Faculty of Applied Science and Engineering at the University of Toronto

CEEA12; Paper 046

Winnipeg, MB; June 17-20, 2012

-5 of $6-$ has made significant strides towards the implementation of the Engineering Graduate Attributes and a continual curriculum improvement process. While the GAC contains a group of dedicated and knowledgeable Faculty members, a challenge remains in building increased capacity across the entire Faculty for the development of assessment tools and measurement of the Attributes. As we move into the permanent data collection and analysis cycle, this will be a priority for our Faculty.

\section{References}

[1] Lorin W. Anderson and David R. Krathwohl. A Taxonomy for Learning, Teaching, and Assessing. New York, Addison Wesley Longman, Inc., 2001.

[2] Susan McCahan, Grant Allen, and Lisa Romkey, "Development of the Graduate Attribute Quality Assurance Process at the University of Toronto," in Proc. CEEA Canadian Engineering Education Conf., CEEC11, Andy Fisher (ed.) (St. John's, NL; 6-8 June 2011), 6 pp., 2011.

[3] Patrick H. Oosthuizen, Urs P. Wyss, Nathalie Kubrick, and Dane Smith, "Benchmarking the Queen's University Mechanical and Materials Engineering Curriculum with the CDIO syllabus,", in Proc. $3^{\text {rd }}$ International CDIO Conference, (MIT, Cambridge, MA, 11-14 June 2007), 12 pp., 2007.

[4] Gloria Rogers, Program Assessment of Student Learning. Blog entry posted May 29, 2010 to http://programassessment.blogspot.com/2010/05/whatis-performance-indicator-anyway.html

\section{Appendix A: University of Toronto Attributes and Global Outcomes}

1. A knowledge base for engineering

1.A. Demonstrate competence in mathematics and modeling.

1.B. Demonstrate competence in natural sciences and engineering fundamentals.

1.C. Demonstrate competence in specialized engineering knowledge appropriate to the program.

2. Problem analysis

2.A. Demonstrate the ability to identify and characterize an engineering problem 
2.B. Demonstrate the ability to formulate a solution plan (methodology) for an engineering problem.

2.C. Demonstrate the ability to formulate and interpret a model.

2.D. Demonstrate the ability to execute solution process for an engineering problem.

3. Investigation

3.A. Demonstrate the ability to define a problem

3.B. Demonstrate the ability to devise and execute a plan to solve a problem

3.C. Demonstrate the ability to use critical analysis to reach valid conclusions supported by the results of the plan

4. Design

4.A. Demonstrate ability to frame a complex, openended problem in engineering terms.

4.B. Demonstrate ability to generate a diverse set of candidate engineering design solutions.

4.C. Demonstrate ability to select candidate engineering design solutions for further development.

4.D. Demonstrate ability to advance an engineering design to a defined end state.

5. Use of engineering tools

5.A. Demonstrate ability to use fundamental modern techniques, resources and engineering tools.

5.B. Demonstrate ability to use discipline specific techniques, resources and engineering tools.

5.C. Show recognition of limitations of the tools used.

6. Individual and team work

6.A. Demonstrate ability to establish and monitor team organizational structure

6.B. Demonstrate ability to promote team effectiveness through individual action

6.C. Demonstrate success in a team based project

7. Communication skills

7.A. Demonstrate the ability to identify and credibly communicate engineering knowledge.

7.B. Demonstrate the ability to use different modes of communication.

7.C. Demonstrate the ability to develop communication through an iterative process.

8. Professionalism:

CEEA12; Paper 046

Winnipeg, MB; June 17-20, 2012

-6 of $6-$
8.A. Demonstrate the ability to describe engineering roles in a broader context, e.g. as pertains to the environment, health, safety, and public welfare

8.B. Demonstrate the ability to recognize the impacts of engineering within a global society (the broader public interest)

8.C. Demonstrate the ability to behave in a professional manner

9. Impact of engineering on society and the environment

9.A. Demonstrate understanding of the relationships among technology and the social, cultural, economic and environmental conditions of society, locally and globally, in both the short-and long-term.

9.B. Demonstrate the ability to identify and choose alternative ways to mitigate or prevent adverse social, environmental, human health and safety impacts.

9.C. Demonstrate awareness of legal issues relevant to an engineering activity.

10. Ethics and equity

10.A. Demonstrate the ability to recognize ethical and equity based dilemmas

10.B. Demonstrate the ability to apply the Code of Ethics and equity principles

10.C. Demonstrate the ability to act ethically and demonstrate individual accountability

11. Economics and project management

11.A. Demonstrate ability to estimate the life-cycle economic and financial costs and benefits for relevant engineering activities

11.B. Demonstrate ability to evaluate the economic and financial performance of an engineering activity and compare alternative proposals on the basis of these measures

11.C. Demonstrate ability to read and understand financial statements for engineering activities

11.D. Demonstrate ability to plan and manage engineering activities to be within time and budget constraints

12. Life-long learning

12.A. Demonstrate the ability to independently summarize, analyze, synthesize and evaluate information from a wide variety of sources (learning independently)

12.B. Demonstrate the ability to develop a strategy to identify and address gaps in knowledge (becoming a self-directed learner) 